\title{
Multiple sclerosis may change how the brain processes sensory information
}

Paula C. Salamone

Sol Esteves

Vladimiro J. Sinay

Indira García-Cordero

Sofía Abrevaya

Blas Couto Federico Adolfi

Miguel Martorell

Agustín Petroni

Adrián Yoris

Kathya Torquati

Florencia Alifano

Agustina Legaz

Fátima P. Cassará

Diana Bruno

Andrew H. Kemp

Eduar Herrera

Adolfo M. García

Agustín lbáñez

Lucas Sedeño

\section{Video Abstract}

Keywords: interoception, interoceptive, exteroception, exteroceptive, multiple sclerosis, neural signatures, sensory processing, bodily signals, neurocognitive, autonomic, heartbeat evoked potential, EEG, MRI, fMRI, insula, anterior cingulate cortex, neurophysiology, neuroanatomy, functional connectivity, body perception

Posted Date: September 20th, 2019

DOI: https://doi.org/10.21203/rs.2.15032/v1

License: (c) (i) This work is licensed under a Creative Commons Attribution 4.0 International License. Read Full License 


\section{Abstract}

Scientists have uncovered new information on how sensory processing can change in multiple sclerosis, or MS. Their findings could open novel avenues for understanding and treating the disease. People with MS typically experience deficits in their ability to smell and taste, abnormal temperature processing, and heightened sensations of pain or fatigue. But there's no clear neurocognitive mechanism to explain such diverse symptoms. A new report in the journal Human Brain Mapping suggests these changes may stem from a problem with interoception. Interoception is a lesser-known skill that helps people feel signals originating from inside of the body - such as the heart beating or the digestive system signaling hunger. Many of the sensory processing issues that characterize MS are associated with brain pathways related to interoception. This link prompted researchers to examine how these pathways are affected in the context of the disease. To accomplish this, the team compared neural activity in people with MS and healthy controls. They tracked the participants' heart rate under two experimental conditions: an exteroceptive task, where participants listened to a recording of a heartbeat and were asked to press a key in synchrony with it, and an interoceptive task, where they tapped the same key while following their own heartbeats. When someone tunes into their own heartbeat, specific electrophysiological changes occur in the brain. These changes are reflected in a parameter known as heartbeat-evoked potential, which can be picked up by EEG. The researchers found that patients with MS showed alterations in this marker of bodysignal processing. Their heartbeat-evoked potential scarcely changed between the two conditions, while the control participants showed clear differences between interoceptive and exteroceptive tasks. To complement these findings, the team used MRI to look at brain structure. They found that the individuals with MS showed degeneration in brain regions associated with interoceptive processing. Functional MRI studies also revealed strong brain connectivity among several key regions in control subjects performing interoceptive tasks. These connections were altered in those affected by MS. Overall, the work indicates that MS may disrupt the ability to process bodily signals. Future investigations into this phenomenon could reveal important new insights into the neurocognitive impact of the disease. 